\title{
QUANTITATIVE ENERGY-FILTERED IMAGE ANALYSIS IN CYTOCHEMISTRY
}

\section{Morphometric analysis of contrast-related images}

\author{
C.W.J. SORBER, A.A.W. DE JONG, N.J. DEN BREEJEN and W.C. DE BRUIJN * \\ AEM Unit, Clinical Pathological Institute I, Erasmus University Rotterdam, P.O. Box 1738, 3000 DR Rotterdam, \\ The Netherlands
}

Received at Editorial Office 18 September 1989; presented at Workshop June 1989

\begin{abstract}
A combination of energy-filtered electron microscopy (EFEM) and an image-analyzing system (IBAS $/ 2000$ ) is used for morphometric analyses of cells and (reaction) products. Image contrast is objectively established and segmentation is based upon intrinsic contrasts, in ultrathin sections. Cross-sectioned platinum-stained erythrocytes are used as a model to determine optimal conditions for constant measuring results for contrast, area and perimeter. Results are related to changes in: (1) the objective-lens diaphragm diameter, (2) three most frequently used contrast modes obtainable by electron spectroscopical imaging (ESI) in a Zeiss EM902 transmission electron microscope (e.g., global, zero loss (or $\Delta E=0 \mathrm{eV}$ ) and $\Delta E=250 \mathrm{eV}$ ), and $(3)$ the number of image integrations $(1-250 \times)$ acquired by real-time video. A thresholding procedure is proposed for objective segmentation of such contrast-related images and applied to measure the area fraction of nuclear chromatin and the diameter of nominal $1 \mathrm{~nm}$ colloidal gold particles.
\end{abstract}

\section{Introduction}

When integrated cytochemical and morphometric image analysis is used to monitor in cells the increase or decrease in product volume and elemental concentration, attention has to be paid to: (a) morphometry, to determine area or area fractions, which are related to the requested volume fraction;

(b) the determination of elemental concentrations in involved items of interest (IoI);

(c) the integration of morphometric information and concentration.

Integrated morphometric/electron probe microanalysis (EPMA) procedures, performed in a scanning transmission electron microscope (STEM), equipped with an energy-dispersive X-ray (EDX) detector, allow an analysis of cytochemical (reaction) products in ultrathin sections [1-5].

Electron energy loss spectroscopy (EELS) has also been combined with STEM instruments. De-

* To whom correspondence should be addressed. dicated STEM/EELS instruments have analyzed the chemical analytical potentials of this combination in biological and non-biological materials [6-23,27]. The formation of elemental distribution images has been described for both combinations $[5,8,13,14,17]$.

In some STEM instruments, the morphometric accuracy is challenged when small, irregularly shaped, reaction products have to be measured $[3-5,8,17,27]$. The IoI to be imaged has to be covered by an array of multiple points during image and signal acquisition by the use of a digital beam-control system. Limitations have been noticed to occur in practice with this type of preacquisition digitization in both morphometric and chemical quantification [5,8]. These limitations are related to:

(a) the large spot size needed to acquire statistically reliable counts for $\mathrm{X}$-ray acquisition (in our case $50 \mathrm{~nm}$, when using a $\mathrm{LaB}_{6}$ cathode [5]);

(b) the dwell time per point $(1-10 \mathrm{~s})$ and the total acquisition time in relation to the number of points required for adequate resolution; 
(c) the complicated on-line deconvolution methods for acquisition of true net-intensity elemental distribution images [3-5,24].

Moreover, specimen tilt, generally required for EDX analysis, interferes with geometric accuracy during morphometric analysis.

The recently introduced energy-filtered electron microscopy (EFEM) $[10-12,25,30,32]$ certainly has not yet proved to overcome all mentioned limitations, but its electron-spectroscopical imaging potentials $[25,26]$ prompted us to investigate its application for a morphometric analysis using:

(1) ESI/TEM images,

(2) EELS/TEM elemental distribution images, and

(3) ESI/TEM and EELS/TEM image integration.

In this paper we will restict ourselves to ESI/ TEM images (1). Items of interest (Iol's) in ESI images will be morphometrically analyzed based upon their intrinsic contrast. Morphometric analyses of such ESI images, after transfer to an image analyzer, will be performed in a type of post-acquisition digitization. Attention will be focused upon two questions: First, how are (objectively determined) image contrasts from the two additional contrast modes (zero loss and $\Delta E=250$ $\mathrm{eV})$ present in the Zeiss EM 902 related to the "classical" global contrast mode present in conventional TEM instruments?

Second, how can ESI contrast variations influence area and perimeter determinations?

The area and perimeter of platinum-stained erythrocyte cross sections will be compared, following application of: (a) the three aforementioned contrast modes, (b) different objective-lens diaphragm diameters and (c) different image-integration cycles for noise reduction. A procedure for objective image segmentation will be proposed to determine areas and area fractions. This procedure will be applied to measure the diameter of nominal $1 \mathrm{~nm}$ colloidal gold particles and chromatin/cell area fractions. In the accompanying paper [31], EELS/TEM element-distribution images (2) will be morphometrically analyzed, and such images will be integrated with ESI/TEM images (3).

\section{Materials and methods}

The instrument used for investigation is a Zeiss EM 902 transmission microscope with an integrated electron energy filter according to Castaing/Henry/Ottensmeyer. Images are recorded on photographic emulsion (SO 163, Kodak, The Hague, NL) or are transferred to an image analyzer (IBAS 2000, Zeiss/Kontron, Oberkochen, FRG) by a TV camera for further analysis, processing or storage by normal routine (Zeiss/Kontron) software.

A schematic cross section of the microscope column and the position of the energy-selecting slit and the spectrometer are given in fig. 1 of ref. [28].

EM 902-derived EFEM allows the acquisition of various ESI images; the three most commonly used types of images, which we used for ultrathin-sectioned material are:

(1) Global images, in which all electrons passing the objective aperture are used for image formation and no energy-selecting slit is installed in the energy-dispersive plane in front of the second projector-lens system.

(2) Zero-loss filtered images, in which primary and elastically scattered electrons passing the objective aperture are used and the inelastically scattered electron are excluded from image formation by the introduction of the energy-selecting slit.

(3) Electron spectroscopic images, which are formed by selecting energy losses with an energyloss width of $20 \mathrm{eV}$. For ultrathin sections, we have chosen $\Delta E=250 \mathrm{eV}$. For optimal imaging of $0.1-0.4 \mu \mathrm{m}$ thick sections, other $\Delta E$ values may be selected.

In the image mode (3), the non-axial ray path of the inelastically scattered electrons is compensated by increasing the high tension $(80000$ $\mathrm{eV})$ with the energy loss involved ( $80250 \mathrm{eV}$ ). This brings the energy band on axis, hence unimpaired image formation can occur.

Materials are derived from:

(a) Mouse virus-infected cell lines, which have been reacted in a pre-embedding mode with a nominal $1 \mathrm{~nm}$ colloidal gold probe, directly con- 
jugated with an antibody (IC5) directed against viral-coat proteins.

(b) Mouse peritoneal resident macrophages (MPrM).

(c) A MPrM cell population which has been reacted with media to detect enzyme activity prior to embedding in Epon. As a consequence, the erythrocytes in that population have acquired a platinum di-amino-benzidine (DAB) complex, which is related to endogenous peroxidase (PO) activity (see for cytochemical procedures refs. [33-37]).

Untreated ultrathin $(20-50 \mathrm{~nm})$ sections from such unstained Epon-embedded materials, collected on unfilmed mesh 400 copper grids, are used for morphometric determinations. The morphometric procedures are applied to images from one of the aforementioned imaging modes (1-3).

As a rule, 100 real-time images are acquired, $(512 \times 512$ pixels $)$ digitized, averaged and stored on disk. To eliminate an inhomogeneous image illumination, a 100-times-integrated, out-of-focus image of the Epon background is also acquired and subtracted from the original image with IBAS subtraction mode 4. After this "shading correction", an eight-bit-deep gray-value frequency histogram is obtained from the image.

The first derivative of this histogram is used to acquire crossovers between the Gaussian curves for image segmentation. Subsequently, guided by the results of this segmentation, a binary image of the IoI, e.g. nucleus or cytoplasm, is obtained. The resultant images are recorded from the IBAS screen by a camera loaded with color film (Ektachrome, Kodak).

By comparing the number of pixels inside binary Iol's with the total number in a cell or frame, the relative area (in pixel\%) could be obtained. Images of a grating replica acquired under the same conditions are used to convert relative to real areas or real lengths. The calibration for the measurements of the $1 \mathrm{~nm}$ gold particles is performed by first calibrating a large population of $10 \mathrm{~nm}$ colloidal gold particles with a grating replica at $50000 \times$ magnification. Subsequently, a sample from the same population was added to the cells bearing the $1 \mathrm{~nm}$ particles. The $10 \mathrm{~nm}$ particles served then as an internal reference.
When necessary, dilatation and/or erosion is used to complete image processing (e.g. to remove remnants of adjacent cells from an image or to convert chromatin area to nuclear area).

\section{Results}

\subsection{Image contrast}

The influence on the image contrast of three different objective-lens diaphragm diameters ( 30 , 60 and $90 \mu \mathrm{m}$, giving apertures of $5,10,20 \mathrm{mrad}$ ), and of three imaging modes (global, zero loss, ESI at $\Delta E=250 \mathrm{eV}$ ) is first objectively established.

As a model, images acquired from the same platinum-containing cross-sectioned and Eponsurrounded erythrocyte are used. One single image of each condition is acquired and analyzed. From this set of 9 images, the grey-value frequency histograms and their first derivatives are acquired. Three such histograms are shown in figs. 1, 2 and 3 . The corresponding images are acquired with a $90 \mu \mathrm{m}$ objective-lens diaphragm, and 100 images are integrated. The image conditions are: global (fig. 1), zero loss (fig. 2) and $\Delta E=250 \mathrm{eV}$ (fig. 3).

The grey values vary from 0 (black) to 255 (white) grey-value units. Two crossover types can be noticed in the first derivatives:

(a) from positive to negative, indicating the top of the Gaussian peaks, and

(b) from negative to positive, indicating the separation between the two Gaussian curves.

When we use the first set of crossovers (a), the contrast between two populations can be defined in integers as the distance between two peaks on the grey-value scale $(\Delta G V)$. Changes in contrast can now objectively be determined. Contrast changes induced by the various imaging modes and objective apertures at $80 \mathrm{keV}$ are shown in table 1:

(1) the global image contrast increases with diaphragm diameter reduction;

(2) the relatively higher zero-loss image contrast is less influenced by diaphragm diameter variation; (3) in the $\Delta E=250 \mathrm{eV}$ images, the (inverted) contrast increases with larger diaphragm diameters. 


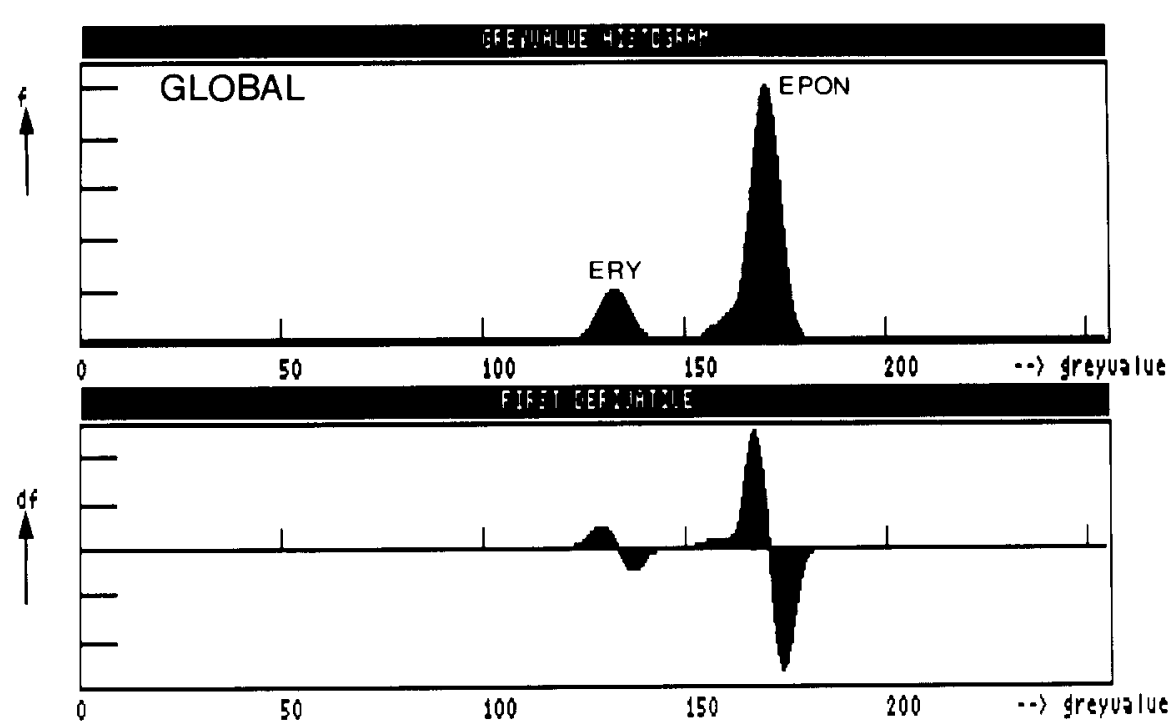

Fig. 1. Grey-value frequency histogram and its first derivative of an erythrocyte taken in the global image mode ( $90 \mu \mathrm{m}$ objective diaphragm).

When the experiments are repeated $(n=5)$ with the same erythrocyte under the same imaging conditions, the instrumental contrast variations, expressed as a coefficient of variance $(\mathrm{CV}=$ standard deviation/mean value $\times 100 \%$ ), appeared to be smaller or equal to $1 \%$ (table 2 ).
The influence upon contrast of the variation in the number of the integrated images $(1 \times$ as compared to $200 \times$ ) is shown in figs. 4 and 5 . In fig. 4 grey-value tracings are shown along a line crossing the erythrocyte. In these images the variation in grey values between a minimum and maximum

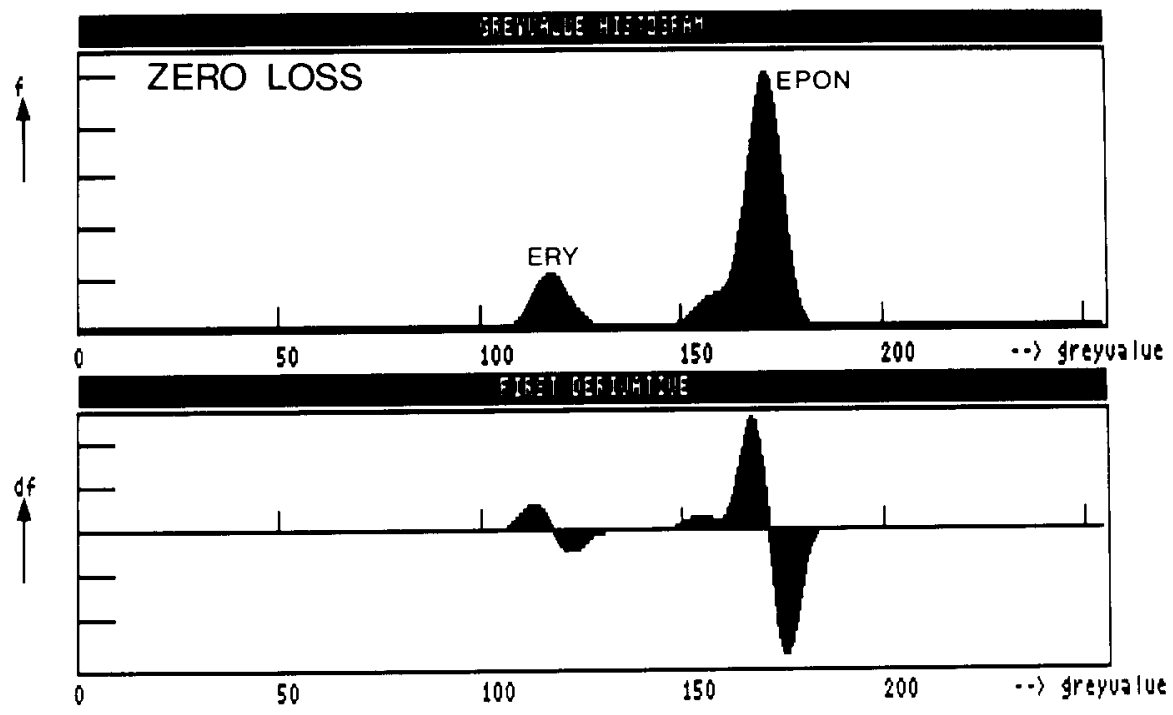

Fig. 2. Grey-value histogram of the same image, under the same conditions in the zero-loss image mode. Note the increase in contrast, defined as the distance between Gaussian maxima ( $\Delta G V$ in fig. 4) 


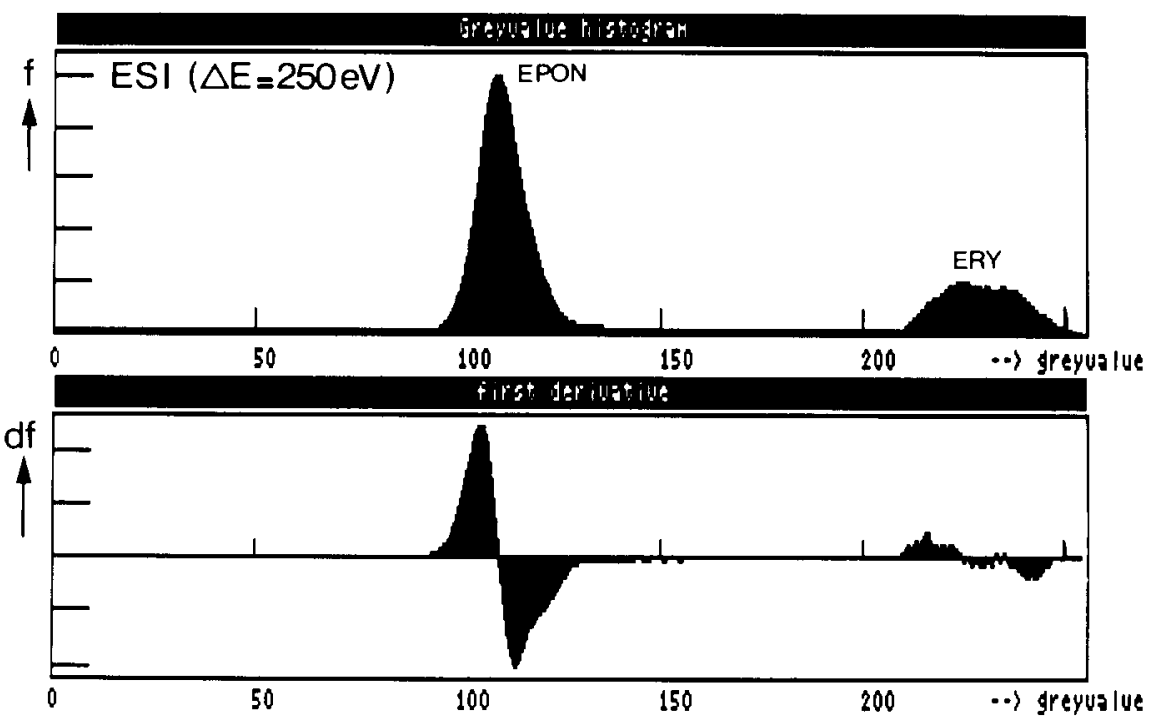

Fig. 3. Grey-value histogram of an erythrocyte in the $\Delta E=250 \mathrm{eV}$ ESI mode ( $90 \mu \mathrm{m}$ objective diaphragm). Note the reversed increased contrast between erythrocyte and Epon.

are indicated for both the Epon surroundings and the erythrocyte. In fig. 5 grey-value histograms of the total images are given. It can be noticed that among other changes the contrast is only slightly influenced by this integration procedure.

In table 3 , the grey values from images integrated once $(1 \times)$ as compared to the mean value of $5 \times, 25 \times, 50 \times 100 \times$ and $250 \times$ are shown. The influence of the various objective apertures upon the contrast of global images at 50 and $80 \mathrm{keV}$ acceleration voltage is investigated. It is noticed that:

(1) the mean contrast is slightly decreased when images are integrated;

(2) reproducibility is slightly changed by multiple image integrations $(\mathrm{CV}=1.5 \%$ as compared to $<1 \%$;

Table 1

Contrast (distance between curves, $\Delta G V$ ) related to image modes and objective-lens diaphragm diameter (one single observation)

\begin{tabular}{llcc}
\hline Image & \multicolumn{3}{l}{$\Delta \mathrm{GV}$ at aperture } \\
\cline { 2 - 4 } & $30 \mu \mathrm{m}$ & $60 \mu \mathrm{m}$ & $90 \mu \mathrm{m}$ \\
\hline Global & 38 & - & 31 \\
$\Delta E=0$ & 54 & 51 & 51 \\
$\Delta E=250$ & 54 & 95 & 101 \\
\hline
\end{tabular}

(3) contrast is increased at lower acceleration voltage;

(4) the observations of table 1 are confirmed.

In table 4, for a different erythrocyte cross section, the same relation is shown for the zero-loss and $\Delta E=250 \mathrm{eV}$ images $(100 \times$ integrated and frequent $(n=5)$ observations) as compared to a single global-image observation:

(1) contrast is uninfluenced by multiple zero-loss observations;

(2) contrast is highly variable by multiple $\Delta E=$ $250 \mathrm{eV}$ observations;

(3) the same trends, as shown in table 1 , for single observations are present.

\subsection{Area}

When we use the second set of crossovers (b), areas occupied by each population can be defined:

Table 2

Contrast related to frequent observations of the same cell (at $80 \mathrm{keV}$ )

\begin{tabular}{|c|c|c|c|c|c|c|c|}
\hline \multirow[t]{2}{*}{ Image } & \multirow[t]{2}{*}{ Integration } & \multicolumn{2}{|c|}{$30 \mu \mathrm{m}$} & \multicolumn{2}{|c|}{$60 \mu \mathrm{m}$} & \multicolumn{2}{|c|}{$90 \mu \mathrm{m}$} \\
\hline & & $X$ & $\mathrm{CV}$ & $X$ & $\mathrm{CV}$ & $x$ & $\mathrm{CV}$ \\
\hline$\Delta E=0$ & $100 \times(n=5)$ & 59.6 & $1.0 \%$ & - & - & - & - \\
\hline
\end{tabular}




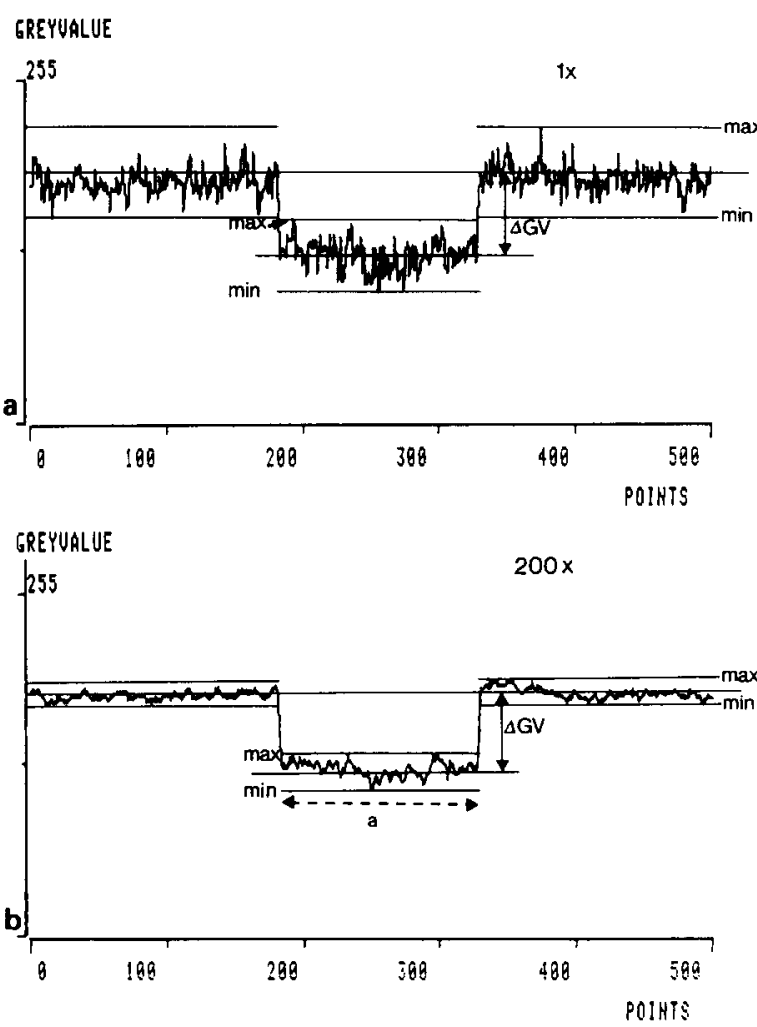

Fig. 4. Contrast tracing through a platinum-containing erythrocyte used for the contrast experiments shown in fig. 2. Grey-value fluctuations are indicated between minimal and maximal values for both the erythrocyte and the Epon surroundings. $\triangle \mathrm{GV}$ is the distance between the two grey-value populations, $a$ the cross section through the erythrocyte. Contrast conditions: zero-loss image $(\Delta E=0 \mathrm{eV}), 90 \mu \mathrm{m}$ objective diaphragm $(1 \times$ and $200 \times$ integration $)$.

number of pixels per Gaussian curve/total pixel number $\times 100$ (area in pixel\%).

Areas of the cross section from one single erythrocyte (shown in table 1) are compared by changing either the objective aperture or the imag-
Table 4

Contrast related to frequent observation of the same cell in the zero-loss $(\Delta E=0 \mathrm{eV})$ or ESI mode $(\Delta E=250 \mathrm{eV})$ as compared to one single observation under global image conditions (at $80 \mathrm{keV}$ )

\begin{tabular}{|c|c|c|c|c|c|c|}
\hline \multirow[t]{2}{*}{ Image } & \multicolumn{2}{|c|}{$30 \mu \mathrm{m}$} & \multicolumn{2}{|c|}{$60 \mu \mathrm{m}$} & \multicolumn{2}{|c|}{$90 \mu \mathrm{m}$} \\
\hline & $x$ & $\mathrm{CV}$ & $X$ & $\mathrm{CV}$ & $x$ & $\mathrm{CV}$ \\
\hline Global & 50 & & 43 & & 40 & \\
\hline$\Delta E=0$ & 63.8 & $<1 \%$ & 60.8 & $<1 \%$ & 64.6 & $<1 \%$ \\
\hline$\Delta E=250$ & 116.0 & $1.7 \%$ & 128.0 & $<1 \%$ & 132.5 & $6.9 \%$ \\
\hline
\end{tabular}

ing mode. Table 5 shows the influences on area measurements:

(1) The calculated erythrocyte area is largest ( 16.8 pixel\%) when measured with the smallest $(30 \mu \mathrm{m})$ diaphragm and smallest (16.1 pixel\%) when using the $60 \mu \mathrm{m}$ diaphragm. At this magnification 1 pixel $\%=232.1 \mathrm{~nm}^{2}$.

(2) The area is not influenced when, with the smallest diaphragm installed, the imaging mode is changed.

(3) With the largest diaphragm installed, the largest variation in area is obtained when the imaging mode is changed.

Variations in integration frequency and objective aperture in relation to erythrocyte area (measured in global images) are shown in table 6:

(1) the area is significantly increased when images are integrated;

(2) the area is hardly influenced by differences in the number of integrations ( $\mathrm{CV}<1.1 \%)$;

(3) the differences in area induced by the diaphragm diameter variations are, at least for these global images, not significant $(1$ pixel $\%=120.2$ $\mathrm{nm}^{2}$ ).

In fig. 5 it can be observed that the number of integrations improves the separation of the two populations.

Table 3

Contrast related to microscope acceleration voltage, integration frequency and diaphragm diameter (global)

\begin{tabular}{|c|c|c|c|c|c|c|c|}
\hline \multirow{2}{*}{$\begin{array}{l}\text { Voltage } \\
\text { (keV) }\end{array}$} & \multirow[t]{2}{*}{ Integration } & \multicolumn{2}{|c|}{$30 \mu \mathrm{m}$} & \multicolumn{2}{|c|}{$60 \mu \mathrm{m}$} & \multicolumn{2}{|c|}{$90 \mu \mathrm{m}$} \\
\hline & & $X$ & $\mathrm{CV}$ & $X$ & $\mathrm{CV}$ & $x$ & $\mathrm{CV}$ \\
\hline 80 & $5 x-250 x$ & 35.3 & $1.5 \%$ & 29.8 & $1.3 \%$ & 26.2 & $1.5 \%$ \\
\hline 50 & $5 \times-250 x$ & 49.8 & $0.9 \%$ & 46.8 & $0.9 \%$ & 47.8 & $0.9 \%$ \\
\hline 50 & $1 \times$ & 51.0 & - & 47.0 & - & 48.0 & - \\
\hline
\end{tabular}




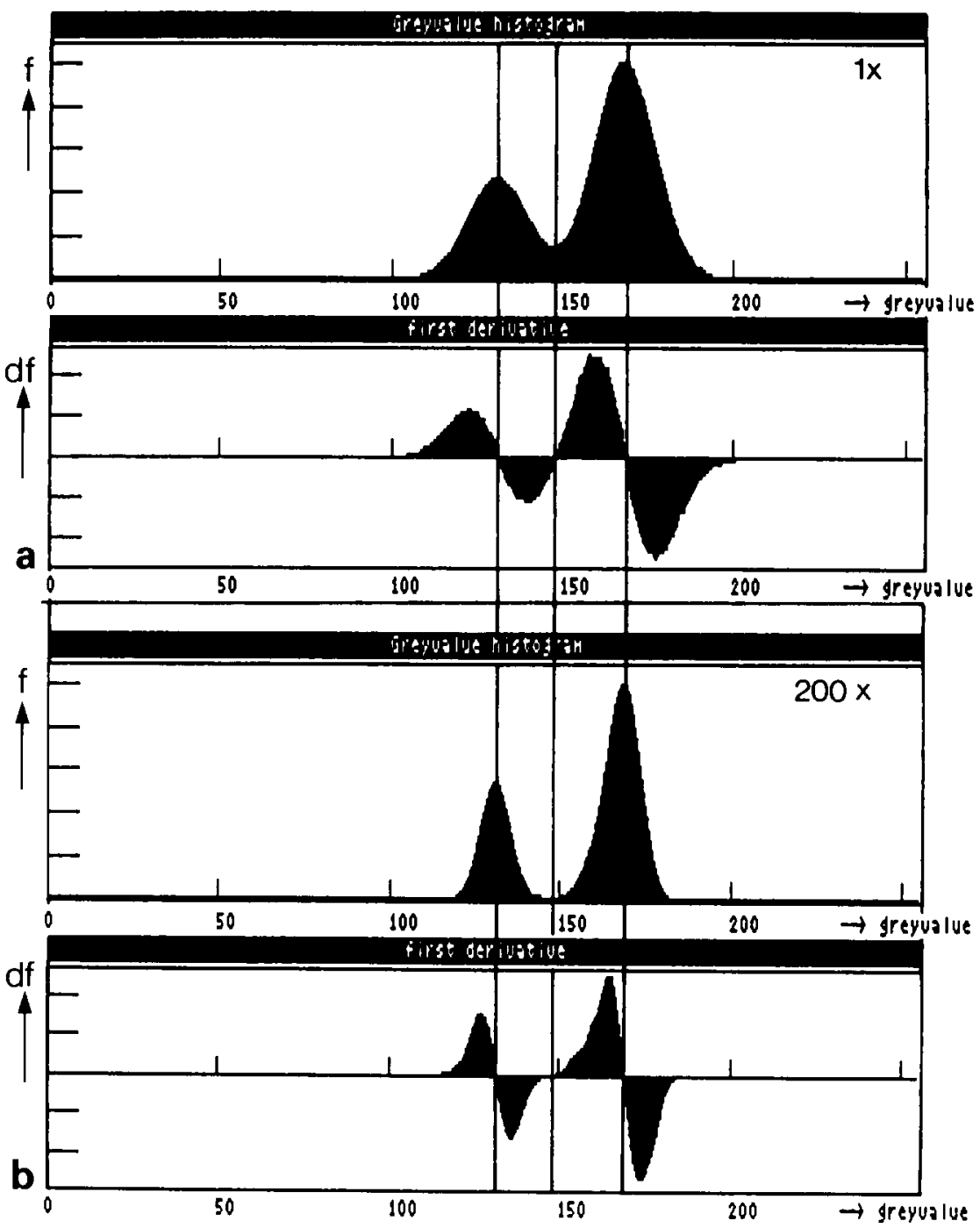

Fig. 5. Grey-value frequency histogram and its first derivative from the same erythrocyte shown in fig. 4. Note the virtual absence of a change in contrast between (a) $(1 \times)$ and (b) $(200 \times)$, and the improved separation between erythrocyte and Epon upon integration.

Table 5

Area related to image modes and objective diaphragm diameter (one single observation)

\begin{tabular}{llcl}
\hline Image & \multicolumn{3}{l}{ Area erythrocyte (pixel\%) } \\
\cline { 2 - 4 } & $30 \mu \mathrm{m}$ & $60 \mu \mathrm{m}$ & $90 \mu \mathrm{m}$ \\
\hline Global & 16.8 & - & 16.7 \\
$\Delta E=0$ & 16.8 & 16.2 & 16.5 \\
$\Delta E=250$ & 16.8 & 16.1 & 16.4 \\
\hline
\end{tabular}

In table 7 , the influence of the same parameters on the area measured in the zero-loss and $\Delta E=$ $250 \mathrm{eV}$ images of the same erythrocyte is shown: (1) Differences in area, among the multiple measurements $(n=5)$ are small $(\mathrm{CV}<1 \%)$.

(2) Differences in area between the various objective apertures for the zero-loss images are small. though significant. 
Table 6

Area (in pixel\%) related to image integration frequency in global images at so keV (the acyuired area differences are mes significant among the various diaphragms)

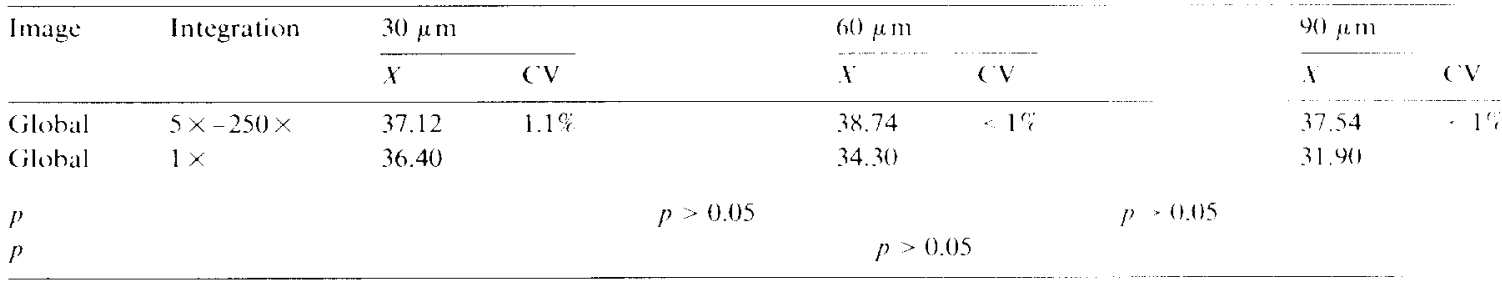

Table 7

Area (in pixel\%) obtained from frequent $(n=5)$ observations of the same cell under zero-loss and l:sl conditions compared lo a single global mode observation (the majority of the areas acyuired are significantly different $(p)$ ( 0.05 ) both among the image moke and the diaphragms selected; $p$ is the significance between area and aperture diameter)

\begin{tabular}{|c|c|c|c|c|c|c|c|}
\hline \multirow[t]{2}{*}{ Image } & \multirow[t]{2}{*}{ Integration } & \multicolumn{2}{|c|}{$30 \mu \mathrm{m}$} & \multicolumn{2}{|l|}{ (ㅇ) $\mu \mathrm{m}$} & \multicolumn{2}{|l|}{$90 \mu \mathrm{m}$} \\
\hline & & $\bar{X}$ & $\mathrm{CV}$ & $X$ & $\mathrm{CV}$ & 1 & $(\mathrm{~V}$ \\
\hline Global & $100 x$ & 28.20 & $(n=1)$ & 27.90 & $(n-1)$ & & \\
\hline$\Delta E=0$ & $100 x$ & 28.32 & $<1 \%$ & 28.04 & $<1 \%$ & 28.42 & $-1 \%$ \\
\hline$\Delta E=250$ & $100 \times$ & 25.35 & $<1 \%$ & 24.80 & $1 \%$ & 28.010 & $.1 \%$ \\
\hline
\end{tabular}

\begin{tabular}{|c|c|c|c|c|c|c|c|}
\hline \multirow[t]{3}{*}{$\Delta E$} & \multirow{3}{*}{$\begin{array}{l}\text { Aperture } \\
(\mu \mathrm{m})\end{array}$} & \multicolumn{6}{|c|}{ Significance $p$} \\
\hline & & \multicolumn{3}{|c|}{$\Delta E=0 \mathrm{cV}$} & \multicolumn{2}{|c|}{$\Delta E-250: V$} & \multirow[b]{2}{*}{901111} \\
\hline & & $30 \mu \mathrm{m}$ & $60 \mu \mathrm{m}$ & $90 \mu \mathrm{m}$ & 30) $\mu \mathrm{m}$ & $60 \mu \mathrm{m}$ & \\
\hline 0 & 30 & - & $<0.05$ & $>0.05$ & $<0.05$ & & \\
\hline 0 & 60 & $<0.05$ &.- & $<0.05$ & & $<0.05$ & \\
\hline 0 & 90 & $>0.05$ & $<0.05$ & & & & $\cdot 0.05$ \\
\hline 251$)$ & 30 & $<0.05$ & - & - & & $\therefore 0.05$ & .19 .015 \\
\hline 250 & 60 & & $<0.05$ & & 0.05 & & 1).015 \\
\hline 250 & 90 & & & $<0.05$ & 0.0 .195 & $\therefore 0.115$ & \\
\hline
\end{tabular}

(3) Differences in area between the various apertures for the $\Delta E=250 \mathrm{eV}$ images are significant. (4) Differences in area measured with zero-loss or $\Delta E=250 \mathrm{eV}$ are significant for each aperture (except for the diaphragms of $30 \mu \mathrm{m}$ versus $90 \mu \mathrm{m}$ for the zero-loss).

(5) The single measurement $(n=1)$ of the global image shows the same trends as in table 5 (1 pixel\% $=120.2 \mathrm{~nm}^{2}$ ).

\subsection{Perimeter}

The same variations related to the erythrocytes relative perimeter are for global images given in table 8 .

Table 8

Perimeter (in pixel\%) related to integration frequency and diaphragm diameter for the global image mode

\begin{tabular}{|c|c|c|c|c|c|c|c|c|}
\hline \multirow[t]{2}{*}{ lmage } & \multirow[t]{2}{*}{ Integration } & \multicolumn{2}{|c|}{$30 \mu \mathrm{m}$} & \multicolumn{2}{|c|}{ 60) $\mu \mathrm{m}$} & & \multicolumn{2}{|c|}{ 9) $\mu \mathrm{m}$} \\
\hline & & $X$ & $\mathrm{CV}$ & $x$ & $(\mathrm{~V}$ & & $x$ & $(V$ \\
\hline Global & $50 x-250 \times$ & 1390 & $1.8 \%$ & 1515 & $3.5 \%$ & & 1467 & $2.2 \%$ \\
\hline Global & $1 \times$ & 2392 & - & 3125 & & & $278 x$ & \\
\hline$p$ & & & & $p$ & & $p-0.05$ & & \\
\hline
\end{tabular}


Table 9

Colloidal gold particle diameter (nominal $1 \mathrm{~nm}$ ) measured at two magnifications

\begin{tabular}{llllll}
\hline$M$ & $\begin{array}{l}D_{\text {circle }} \\
(\mathrm{nm})\end{array}$ & $\sigma$ & $n$ & $N_{\text {pix } / \text { part }}$ & $\begin{array}{l}\text { Shape- } \\
\text { factor }\end{array}$ \\
\hline $225000 \times$ & 1.67 & 0.26 & 48 & 25 & 0.57 \\
$400000 \times$ & 1.32 & 0.35 & 25 & 50 & 0.38 \\
\hline
\end{tabular}

$80 \mathrm{keV}, \alpha=90 \mu \mathrm{m}$, zero loss $(\Delta E=0 \mathrm{eV}), 20 \times$ integration.

(1) The perimeter is highly influenced by the differences in integration.

(2) The perimeter decreases with increasing integrations and stabilizes after about $50 \times(\mathrm{CV}=$ $2 \%-4 \%)$.

(3) The perimeter differences for different apertures and this image mode are significant (except for the diaphragms of $60 \mu \mathrm{m}$ versus $90 \mu \mathrm{m}$ ).

In conclusion:

(1) A $100 \times$ image integration seems to be a fair compromise between speed and noise reduction for both area and perimeter.

(2) Different objective apertures induce significant area differences.

(3) Different image modes (zero-loss or $\Delta E=250$ $\mathrm{eV}$ ) induce small though significant variations in the areas measured.

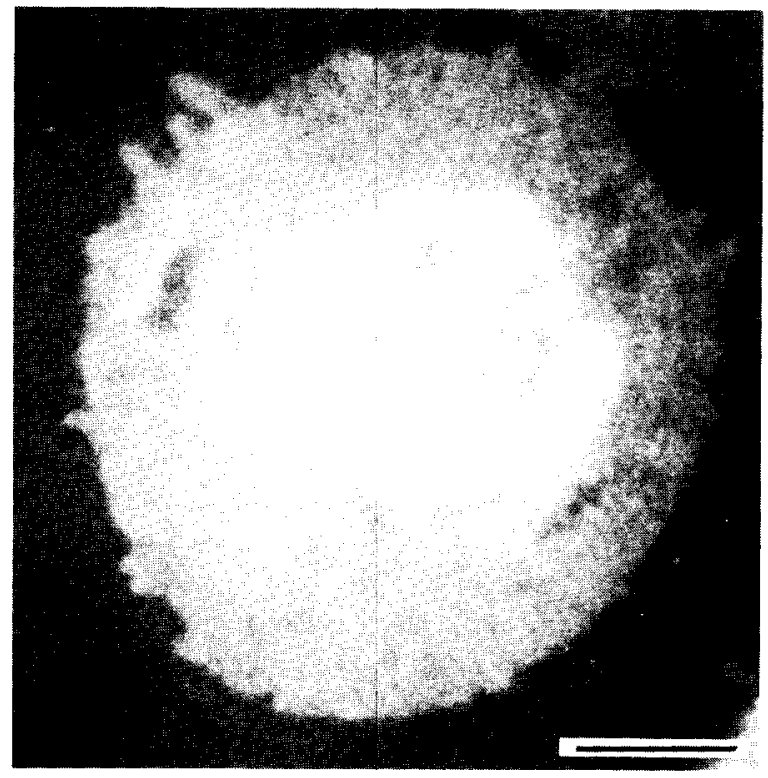

Fig. 6. Unstained ultrathin-sectioned MPrM cell. Imaging condition $\Delta E=250 \mathrm{eV}$ and $90 \mu \mathrm{m}$ objective diaphragm, showing the intrinsic contrast in the bright nuclear chromatin. Bar $=1$ $\mu \mathrm{m}$.

At increased $\Delta E$ values, the reduction in beam intensity favours the choice of the $90 \mu \mathrm{m}$ objective-lens diaphragm. As a consequence, a $90 \mu \mathrm{m}$ objective-lens diaphragm has been used in two

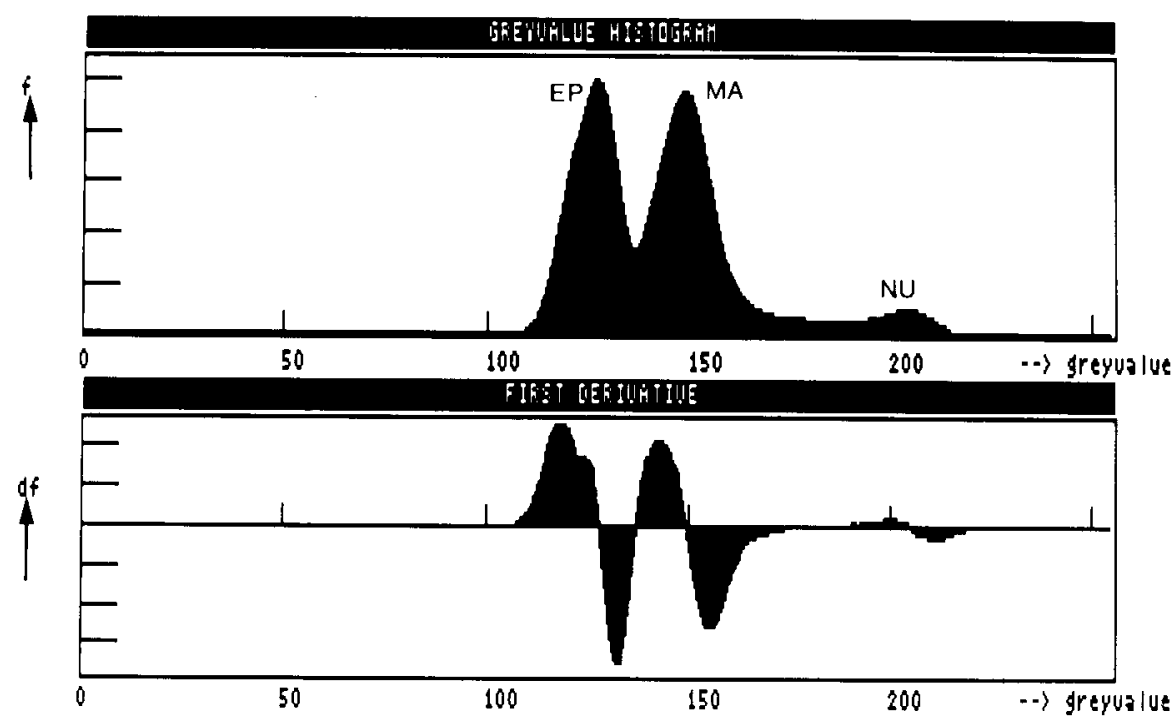

Fig. 7. Grey-value frequency histogram of MPrM cell, showing three grey-value populations, Epon/cytoplasm/nuclear chromatin. The binary images are shown in the color plate fig. 8 , panels a and $b$. 

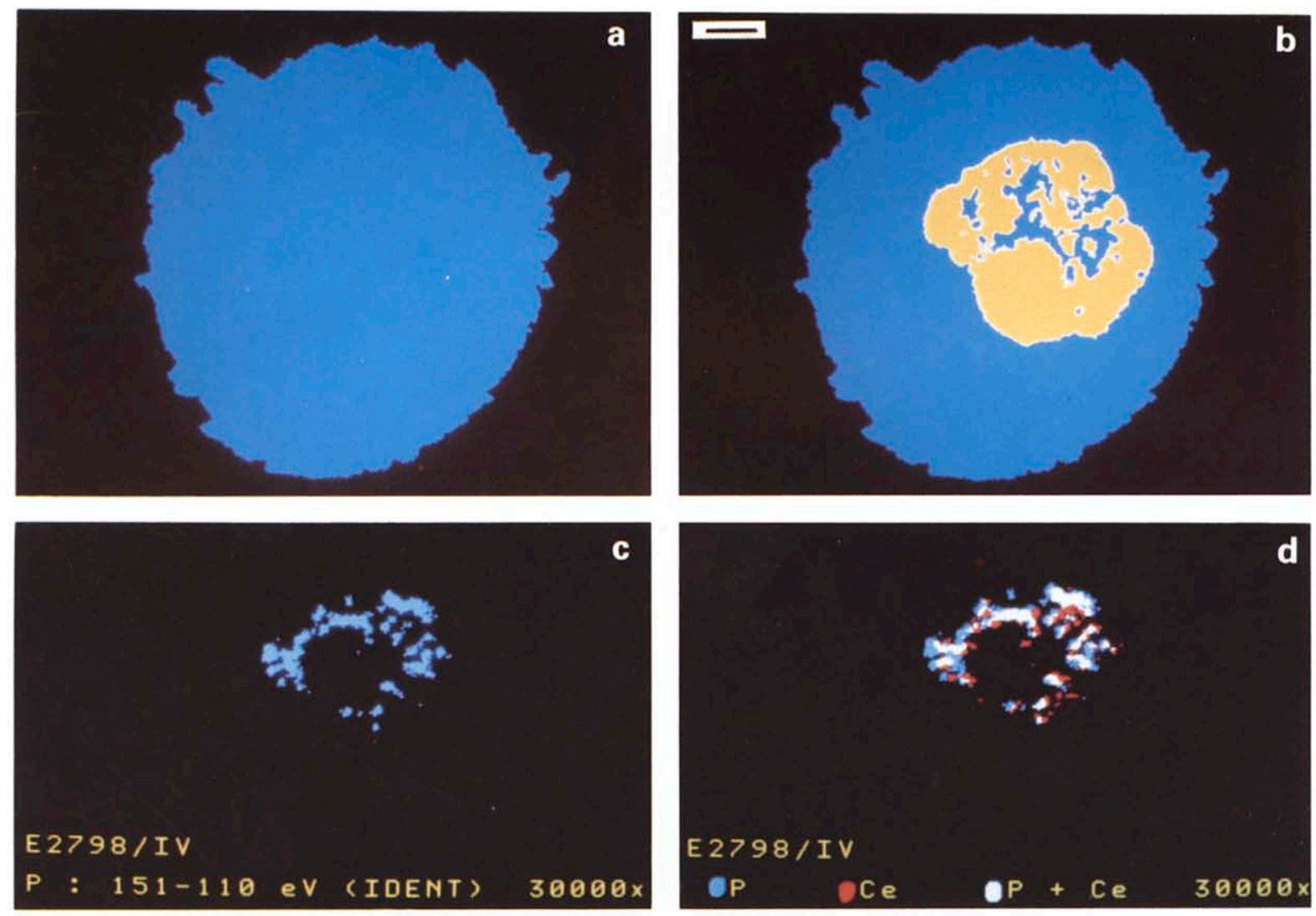

E2798/IV

$P$ : $151-110 \mathrm{eV}$ (IDENT) $30000 \mathrm{x}$

Q

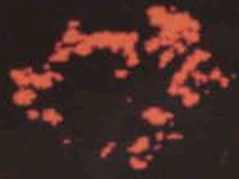

E2798/IV

Ce : $903-863$ eV (IDENT) $30000 x$
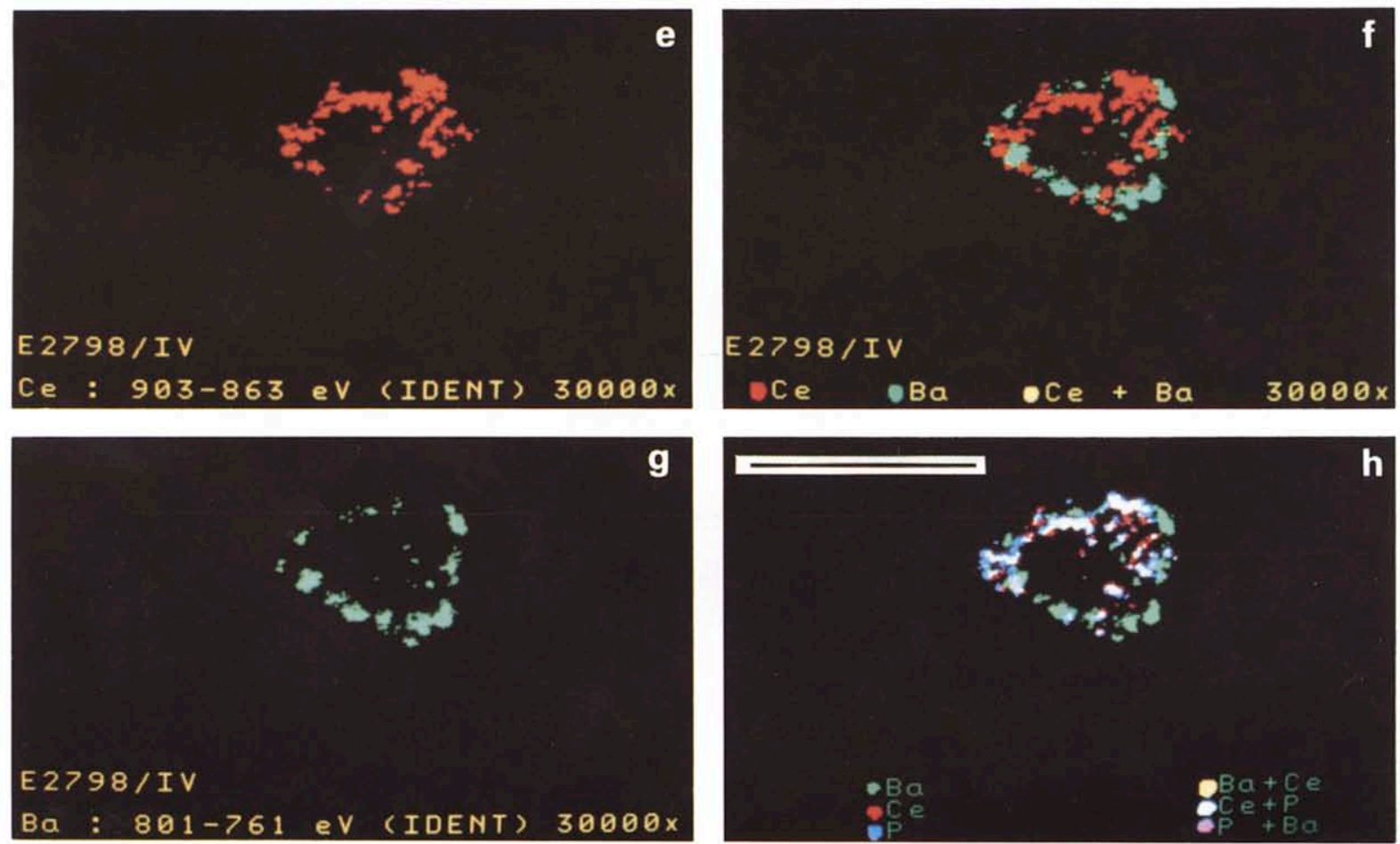
experiments performed to measure Iol's based upon their intrinsic contrast and after application of the proposed objective segmentation procedure.

In table 9, results are collected from measurements of $1 \mathrm{~nm}$ colloidal gold particles. From the same microscope fields at two magnifications. zero-loss images are acquired at two different magnifications to monitor the accuracy of the measurements. Hence, two different mean diameters $(1.32$ and $1.67 \mathrm{~nm})$ are calculated and two different numbers of pixels/particle and shape factors are acquired.

In figs. 6-8 images and a histogram are shown from an experiment to obtain the area fraction of the chromatin in an unstained nucleated MPrM cell.

The grey-value frequency histogram of the $\Delta E$ $=250 \mathrm{eV}$ image shown in fig. 6 is given in fig. 7. In this frequency histogram three populations are present: (1) Epon. (2) cytoplasm. (3) nuclear chromatin.

The nuclear-chromatin population has in this case the highest grey value. To separate these three populations, crossovers (type b) between three Gaussian-shaped distributions are found by the first derivative, obtained from the histogram fig 7. The images are made binary by two thresholds positioned at the two selected crossovers, thus segmenting the image in cell/Epon (fig. 8a) and nuclear chromatin/cytoplasm (fig. 8b). Binary images are used to calculate relative cytoplasmic and nuclear chromatin areas, and hence the area fraction of the nuclear chromatin (0.17). Alternatively, when a dilatation step is used to "fill-in" the holes in the nucleus, the area fraction nucleus/cytoplasm is obtained (0.22). Prior to measuring. we used an erosion step to remove parts of the neighbouring cells in the corners.

\section{Discussion}

The aim for a correct segmentation of electron microscopic images is intimately related to the image contrast. When intrinsic cell organelles are to be separated and staining has to be avoided, instrumental contrast manipulation is the first choice for optimal results. The answer to this problem in dedicated STEM instruments was ratio contrast [19]. Zero-loss and ESI-contrast has become an alternative in TEM instruments $[25,38$ 40]. The announced contrast improvements [25] are confirmed in this paper by objective measurements.

The consequences of the choice of the image mode, the integration frequency and objective aperture are considered in their effects upon two morphometric parameters: area and perimeter.

However, the nature of the intrinsic contrast is enclosed in the chemical composition of cellular material visualized. The endogenous contrast between the nuclear chromatin and the cytoplasm as shown at $\Delta E=250 \mathrm{eV}$ might be induced by several possible factors: (a) the chromatin versus cytoplasmic phosphorus concentration; (b) the spectral position of the phosphorus $L_{1.2}$ edge below the carbon $\mathrm{K}$ edge at $\Delta E=286 \mathrm{eV}$ and (c) to a lesser degree, the section thickness. Moreover, Carlemalm et al. [41] indicated that the difference in hydrogen concentration might play a dominant role. Section thickness has not successfully been determined in our material. Methods to determine objectively layer/section thickness have become available [42-45]. However, these methods need. in our hands, adaptation for extremely thin, unfilmed sections of biological material due to the virtual absence of a low loss edge.

Once an averaged shading-corrected ESI image

Figs. 8a-8b. (a) Binary image of MPrM cell, objectively segmented according to the frequency histogram in fig. 7. (b) Binary image of the nuclear chromatin of the same cell, segmented according to fig. 7. Bar indicates $1 \mu \mathrm{m}$ (also pertaining 10 (a)).

Figs. 8c 8h. Images described in the accompanying paper [31]. (c) Phosphorus semi-net-intensity element-distribution image of a lysosome, in which three lysosomal enzymes will he differentiated. (d) Superposition of the ceriun and phosphorus leading to a new white color at the places where the two images coincide. (e) Cerium semi-net-intensity element-distribution image of the same lysosome. (f) Superposition of cerium and barium net-intensity images showing the virtual absence of the new (yellow) color at the places of coincidence. (g) Barium semi-net-intensity element-distribution image of the same lysosome. (h) Superposition of three net-intensity element-distribution images, showing the slight coincidence of barium and phosphorus as a new color at a few places. Bar pertaining to $(c)-(h)$ indicates $550 \mathrm{~nm} ;(a)-(h)$ are pseudo-colored images. 
is acquired. objective threshold determination is the next step. Conversion of the image into the frequency domain is generally advocated [24.46 48]. Although several alternative methods for segmentation are available [48-51], we have not used other segmentation methods. The first derivativebased method we used is reproducible and fast. but sometimes requires a (median or moving boxcar filter) smoothing step to eliminate additional unwanted minor crossovers [24]. Other methods proposed [45-51], which may give more precise results, still have to be investigated.

Once the Gaussian curves are segmented, relative areas (pixel\%) or area ratios can directly be measured, or relative areas can be converted to real areas. Limitations encountered with our procedure are:

(1) When lol grey-values are present in low numbers and hence a small Gaussian curve is present. it is hard to detect the crossovers because of grey-value overlap in the frequency histogram. A solution for this problem can be a larger initial magnification or a post-magnification step in the IBAS, or a segmentation procedure based upon the "background" population alone (mean + 13 5) $\times$ standard deviation).

(2) When the contrast between Iol and its surroundings is too small, two peaks will overlap. which makes it impossible to separate populations completely.

(3) When images are noisy, pixels which do not belong to the Iol are segmented as well.

There might also be a difference in the degree of confidence in the delineation of the Iol's present in the final image [52]. When in our first derivative-based method Gaussian peaks are completely separated (figs. 1-3), the degree of confidence of segmentation is unimpaired. Confidence is challenged when two curves overlap and the crossover indicates the lowest value in between the two (fig. 7. cytoplasm/Epon).

Another aspect is the degree of accuracy of the acquired areas, induced by (the choice of) the number of pixels per particle for the area determination of very small particles, as demonstrated by our measurements of the colloidal-gold particles shown in table 9. Theoretical aspects of this kind of inaccuracy are well known [53].
When staining is permitted (performed) the choice of stain is important. It has to be realized that most of the staining strategies in the past intended to increase the elastic scatter. Recently Reimer et al. [28 30$]$ have calculated the gain in contrast between global and zero-loss images using. among other examples. $100 \%$ evaporated platinum films on pure carbon films. In this paper we used platinum-containing erythrocytes for similar contrast experiments. From such erythrocytes, from the same Fpon block as we used here, we have calculated earlier with EPMA a platinum weight percentage of $46 \%$.

Once an optimal contrast condition is obtained. noise reduction and shading correction are the next steps to consider. Although these steps are vital for a good final result. we just followed the (Zeiss/IBAS/Kontron) instructions as described and selected initially an averaging of 100 images. The noise/eontrast aspect related to objective decisions in segmentation is outlined in fig. 4 , by at grey-value cross section through the erythrocite used for the contrast experiments alter $1 \times$ and $200 \times$ averaging. The horizontal lines indicate the mean (residual) grey value in each population (Epon/erythrocyte). The residual texture (norise or knife marks) in each population is indicated by the original tracing. The width of the erythrocyce $(\langle a\rangle)$ as determined from this cross section depends on whether the highest (max) or the lowest points (min) of the noise curves are interconnected. Various solutions to this problem have been proposed, e.g. linear and non-linear laplace filtering [46,47].

Recently. Verbeek et al. $150 \mid$ proposed the use of a $\mathrm{min} / \max$ filter, which located $\langle a\rangle$ halfway between $\langle\Delta G V\rangle$ and midway between the (min) and $(\max )$ noise curves at that height. (Our decisions for segmentation in noise-reduced images are in principle similar (halfway between the two (Gaussian peaks). It has to be realised that our choice to use erythrocyte cross sections as an object for segmentation reduced the risk of losing high resolution information due to image integration. When high resolution aspects in the images are to be expected and have to be preserved other strategies have to be chosen (see ref. [52]).

The use of $90 \mu \mathrm{m}$ objective-fens diapluragm 
during ESI analysis is chosen for brightness reasons, but includes the risk of significantly different area values to be obtained. This brings us to the semantic question: which value will represent the true value? Future experiments on specimens with externally verifiable values might bring the answer to the question about the degree of systemic error which is present in our (mainly global) observations.

In conclusion, the following statements can be made:

I. Image contrast analysis.

(a) The first derivative of the frequency histogram of ESI images allows an objective determination of the image-contrast values present in $46 \mathrm{wt} \%$ platinum-stained erythrocytes and $100 \%$ colloidal-gold particles.

(b) The contrast improvement of the two EFEM imaging modes used in the Zeiss EM 902 (zero-loss and $\Delta E=250 \mathrm{eV}$ ) are objectively established and compared with the classical global TEM contrast mode.

II. Morphometric analysis.

(a) A method based upon the first derivative of the image frequency histogram is also used for objective segmentation. By that procedure, cell components can be segmented by their intrinsic contrast.

(b) The success of objective segmentation is limited by small differences in intrinsic contrast between Iol and surroundings, the low occurrence of the Iol and the high noise in the original image.

(c) It is possible to get area fractions from ESI images from unstained, only aldehyde-fixed cells by energy-filtering with $\Delta E=250 \mathrm{eV}$ electrons when these conditions are fulfilled.

(d) Significantly different area and perimeter values are obtained with various objective-lens diaphragms and image modes.

\section{References}

[I] W.C. de Bruijn and J.Ph. Zeelen, Beitr. Elektronemikrosk. Direktabb. Oberfl. 16 (1983) 385.

[2] W.C. de Bruijn, in: Scanning Electron Microscopy/1985, Fd. O. Johari (SEM, AMF O'Hare, IL. 1985) p. 697.

[3] W.C. de Bruijn and M.I. Cleton-Soeteman, in: Scanning
Electron Microscopy/1985. Ed. O. Johari (SEM, AMF O'Hare. IL. 1985) p. 715.

[4] M.I. Cleton, J.M. Roelofs. C.J.G. Blok-Van Hoek and W.C. de Bruijn, in: Scanning Electron Microscopy/1986 Ed. O. Johari (SEM, AMF O'Hare, IL, 1986) p. 999.

[5] W.C. de Bruijn, H.K. Koerten, M.I. Cleton-Soeteman and C.J.G, Blok-Van Hoek, in: Scanning Electron Microscopy/1987, Ed. O. Johari (SEM, AMF O'Hare. IL, 1987) p. 1651

[6] C. Colliex. C. Jeanguillaume and C. Mory. J. Ultrastruct. Res. 88 (1984) 177.

[7] C. Colliex, Ultramicroscopy 18 (1985) 131.

[8] C. Colliex, T. Manoubi and O.L. Krivanek, J. Electron Microsc. 35 (1986) 313.

[9] A. Engel and R. Reichelt, J. Ultrastruct. Res. 88 (1984) 105 .

[10] K.M. Adamson-Sharpe and F.P. Ottensmeyer, J. Microscopy 122 (1981) 309.

[11] A.L. Arsenault and F.P. Ottensmeyer, Proc. Natl. Acad. Sci. USA 80 (1983) 1322.

[12] A.L. Arsenault and F.P. Ottensmeyer, J. Microscopy 133 (1984) 69

[13] G. Harauz and F.P. Ottensmeyer, Ultramicroscopy 12 (1984) 309.

[14] G. Harauz and F.P. Ottensmeyer, Science 226 (1984) 936.

[15] R.D. Leapman, C.E. Fiori, K.E. Gorlen, C.C. Gibson and C.R. Swyt. Ultramicroscopy 12 (1984) 281.

[16] R.D. Leapman, K.E. Gorlen and R.E. Swyt, in: Scanning Electron Microscopy/1985. Ed. O. Johari (SEM. AMF O'Hare, IL. 1985) p. 1.

[17] P.F. Ottensmeyer, J. Ultrastruct. Res. 88 (1984) 121

[18] H. Shuman and A.P. Somlyo, Proc. Natl. Acad. Sci. USA 79 (1982) 106.

[19] H. Shuman, C.F. Chang, and A.P. Somlyo. Ultramicroscopy 19 (1986) 121.

[20] H. Shuman and A.P. Somlyo, Ultramicroscopy 21 (1987) 23.

[21] A.P. Somlyo and H. Shuman, Ultramicroscopy 8 (1982) 219.

[22] A.P. Somlyo, J. Ultrastruct. Res. 88 (1984) 135.

[23] N.I. Zaluzec, Ultramicroscopy 18 (1985) 185.

[24] W.C. de Bruijn and M.P.C. van Miert. in: Scanning Electron Microscopy/1988. Ed. O. Johari (SEM, AMF O'Hare, IL, 1988) p. 319.

[25] U.B. Hezel, R. Bauer, E. Zellmann and W.I. Miller, in: Proc. 44th Annual EMSA Meeting. Ed. G.W. Bailey (San Francisco Press, San Francisco, 1988) p. 68.

[26] U.B. Hezel. R. Bauer and D. Meissner, in: Proc. Congr. on Electron Microscopy. Bologna, 1987. p. 267.

[27] R.F. Egerton and P.A. Crozier, J. Microscopy 148 (1987) 157.

[28] L. Reimer. I. Fromm and R. Rennekamp, Ultramicroscopy 24 (1988) 339.

[29] L. Reimer and M. Ross-Messemer. J. Microscopy 155 (1989) 169.

[30] L. Reimer, I. Fromm, R. Rennekamp and M. Ross-Messemer, in: Proc. VII Conferencja Mikrokopii Electronowej Ciala Stalego, Krakow. 1989, p. 29 
[31] C.W.J. Sorber. J.B. van Dort, P.C. Ringeling, M.I. Cleton Soeteman and W.C. de Bruijn, Ultramicroscopy 32 (1990) 69.

[32] M.I. Cleton, L.J. Mostert. C.W.J. Sorber, A.A.W. de Jong. C.M.H. de Jeu-Jaspars and W.C. de Bruijn, Cell Tissue Res. 256 (1989) 601.

[33] W.C. de Bruijn, J. van der Meulen, P. Brederoo and W.Th Daems, Histochemistry 84 (1986) 492

[34] J.B. van Dort, J.Ph. Zeelen and W.C. de Bruijn, Histochemistry 87 (1987) 71

[35] J.B. van Dort. (j.A.M. Ketelaars. A.A.W. de Jong and W.C. de Bruijn, Inst. Phys. Conf. Ser. 93 (1988) 577.

[36] J.B. van Dort. G.A.M. Ketelaars, W.Th Daems and W.C. de Bruijn. Histochemistry 92 (1989) 243.

[37] L.J. Mostert, M.I. Cleton, W.C. de Bruijn, J.F. Koster and H.G. van Eijk, Int. J. Biochem. 21 (1989) 39.

[38] W.C. de Bruijn, C.W.J. Sorber, A.A.W. de Jong and M.I. Cleton-Soeteman, in: Proc. 1st Eur. Workshop Fur. Microbeam Analysis Soc. 1989. p. 191.

[39] L.D. Peachy, J.P. Heath and G. Lamprecht, in: Proc. 44th Annual EMSA Meeting. Ed. G.W. Bailey (San Francisco Press, San Francisco, 1988) p.88

[40] J. Trinick and J. Berriman, Ultramicroscopy 21 (1987) 393.
141] I. Carlemalm, J.D. Acetarin. W. Villiger, C. Colliex and F. Kellenberger, J. Ultrastruct. Res. 80 (1984) 3.39.

[42] J. Berriman and K. R. Leonard. Ultramicroscopy 19) (1986 349.

[43] R.D. Leapman, C.E. Fiori and C.R. Swyt, J. Microscopy 133 (1984) 239.

[44] D.R. I.iu and L.M. Brown. J. Microscopy 147 (1987) 37

[45] T. Malis, S.C. Cheng and R.F. Fgerton. I. Flectron Microsc. Tech. 8 (1988) 193

[46] J. Russ and J. Russ. J. Microscopy 135 (1984) 89.

[47] H.J.G. Gundersen. J. Microscopy 143 (1986) 3

[48] J. Russ and J. Russ. J. Microscopy 148 (1987) 26.3

[49] G. Johannsen and J. Bille, in: Proc. Int. Conf. on I'attern Recognition. Inst. Flectrical Flectronic Fngineers $(6 \mathrm{~cm}$ puter Soc. Press. München. 1982) p. 140.

[50] P.W. Verbeek. H.A. Vrooman and L.I. van Vliet. Signal Processing 15 (1988) 249

[51] F. Meyer, Biol. Cell. 41 (1981) l.

152] P. Trebbia, in: Proc. 1st Eur. Workshop. Eur. Microheam Analysis Soc. 1989. p. 107.

[53] I.T. Young, Anal. Quant. Cytol. Histol. 10 (1988) 269. 